ANNALES

POLONICI MATHEMATICI

$99.2(2010)$

\title{
On para-Nordenian structures
}

\author{
by Arif A. SAlimov (Erzurum and Baku) and Filiz AgCA (Trabzon)
}

\begin{abstract}
The aim of this paper is to investigate para-Nordenian properties of the Sasakian metrics in the cotangent bundle.
\end{abstract}

1. Introduction. Let $\left(M^{n}, g\right)$ be an $n$-dimensional Riemannian manifold, $T^{*} M^{n}$ its cotangent bundle and $\pi$ the natural projection $T^{*} M^{n} \rightarrow M^{n}$. A system of local coordinates $\left(U, x^{i}\right), i=1, \ldots, n$ on $M^{n}$ induces on $T^{*} M^{n}$ a system of local coordinates $\left(\pi^{-1}(U), x^{i}, x^{\bar{\imath}}=p_{i}\right), \bar{\imath}:=n+i(\bar{\imath}=1, \ldots, 2 n)$, where $x^{\bar{\imath}}=p_{i}$ are the components of the covector $p$ in each cotangent space $T_{x}^{*} M^{n}, x \in U$, with respect to the natural coframe $\left\{d x^{i}\right\}, i=1, \ldots, n$.

We denote by $\Im_{s}^{r}\left(M^{n}\right)$ (resp. $\Im_{s}^{r}\left(T^{*} M^{n}\right)$ ) the module over $F\left(M^{n}\right)$ (resp. $\left.F\left(T^{*} M^{n}\right)\right)$ of $C^{\infty}$ tensor fields of type $(r, s)$, where $F\left(M^{n}\right)\left(\right.$ resp. $\left.F\left(T^{*} M^{n}\right)\right)$ is the ring of real-valued $C^{\infty}$ functions on $M^{n}$ (resp. $\left.T^{*} M^{n}\right)$.

Let $X=X^{i} \frac{\partial}{\partial x^{i}}$ and $\omega=\omega_{i} d x^{i}$ be the local expressions in $U \subset M^{n}$ of a vector and a covector (1-form) field $X \in \Im_{0}^{1}\left(M^{n}\right)$ and $\omega \in \Im_{1}^{0}\left(M^{n}\right)$, respectively. Then the complete and horizontal lifts ${ }^{C} X,{ }^{H} X \in \Im_{0}^{1}\left(T^{*} M^{n}\right)$ of $X \in \Im_{0}^{1}\left(M^{n}\right)$ and the vertical lift ${ }^{V} \omega \in \Im_{0}^{1}\left(T^{*} M^{n}\right)$ of $\omega \in \Im_{1}^{0}\left(M^{n}\right)$ are given, respectively, by

$$
\begin{aligned}
{ }^{C} X & =X^{i} \frac{\partial}{\partial x^{i}}-\sum_{i} p_{h} \partial_{i} X^{h} \frac{\partial}{\partial x^{\bar{\imath}}} \\
{ }^{H} X & =X^{i} \frac{\partial}{\partial x^{i}}+\sum_{i} p_{h} \Gamma_{i j}^{h} X^{j} \frac{\partial}{\partial x^{\bar{\imath}}} \\
{ }^{V_{\omega}} & =\sum_{i} \omega_{i} \frac{\partial}{\partial x^{\bar{\imath}}}
\end{aligned}
$$

with respect to the natural frame $\left\{\frac{\partial}{\partial x^{i}}, \frac{\partial}{\partial x^{i}}\right\}$, where $\Gamma_{i j}^{h}$ are the components of the Levi-Civita connection $\nabla_{g}$ on $M^{n}$ (see [11] for more details).

2010 Mathematics Subject Classification: Primary 53C07; Secondary 53C15.

Key words and phrases: Sasakian metric, cotangent bundle, vertical and horizontal lift, para-Nordenian metric. 
For each $x \in M^{n}$ the scalar product $g^{-1}=\left(g^{i j}\right)$ is defined on the cotangent space $\pi^{-1}(x)=T_{x}^{*}\left(M^{n}\right)$ by

$$
g^{-1}(\omega, \theta)=g^{i j} \omega_{i} \theta_{j}
$$

for all $\omega, \theta \in \Im_{1}^{0}\left(M^{n}\right)$.

A Sasakian metric ${ }^{S} g$ is defined on $T^{*} M^{n}$ by the following three equations

$$
\begin{aligned}
{ }^{S} g\left({ }^{V} \omega,{ }^{V} \theta\right) & ={ }^{V}\left(g^{-1}(\omega, \theta)\right)=g^{-1}(\omega, \theta) \circ \pi, \\
{ }^{S} g\left({ }^{V} \omega,{ }^{H} Y\right) & =0 \\
{ }^{S} g\left({ }^{H} X,{ }^{H} Y\right) & ={ }^{V}(g(X, Y))=g(X, Y) \circ \pi
\end{aligned}
$$

for any $X, Y \in \Im_{0}^{1}\left(M^{n}\right)$ and $\omega, \theta \in \Im_{1}^{0}\left(M^{n}\right)$. Since any tensor field of type $(0,2)$ on $T^{*} M^{n}$ is completely determined by its action on vector fields of type ${ }^{H} X$ and ${ }^{V} \omega$ (see [11, p. 280]), it follows that ${ }^{S} g$ is completely determined by (1.4)-(1.6).

From (1.1) and 1.2 we notice that the complete lift ${ }^{C} X$ of $X \in \Im_{0}^{1}\left(M^{n}\right)$ is expressed by

$$
{ }^{C} X={ }^{H} X-{ }^{V}(p(\nabla X)),
$$

where $p(\nabla X)=p_{i}\left(\nabla_{h} X^{i}\right) d x^{h}$.

Using (1.4)-1.7), we have

$$
{ }^{S} g\left({ }^{C} X,{ }^{C} Y\right)={ }^{V}(g(X, Y))+{ }^{V}\left(g^{-1}(p(\nabla X), p(\nabla Y))\right),
$$

where $g^{-1}(p(\nabla X), p(\nabla Y))=g^{i j}\left(p_{l} \nabla_{i} X^{l}\right)\left(p_{k} \nabla_{j} Y^{k}\right)$.

Since the tensor field ${ }^{S_{g}} \in \Im_{2}^{0}\left(T^{*} M^{n}\right)$ is also completely determined by its action on vector fields of type ${ }^{C} X$ and ${ }^{C} Y$ (see [11, p. 237]), we have an alternative characterization of ${ }^{S} g$ : a Sasakian metric ${ }^{S} g$ on $T^{*} M^{n}$ is completely determined by the condition (1.8).

Sasakian metrics on the tangent bundle were introduced in [9] by the Japanese geometer S. Sasaki. Sasakian metrics (diagonal lifts of metrics) on tangent bundles were also studied in [3], 11]. In the more general case of tensor bundles of type $(1, q),(0, q)$ and $(p, q)$, Sasakian metrics and their geodesics were considered in [1], 6], 7]. Sasakian metrics on the frame bundle were first considered by K. P. Mok [5] (see [2] for more details). This paper is concerned with para-Nordenian properties of the Sasakian metric on the cotangent bundle.

2. Levi-Civita connection of ${ }^{S} g$. On $U \subset M^{n}$, we put

$$
X_{(i)}=\frac{\partial}{\partial x^{i}}, \quad \theta^{(i)}=d x^{i}, \quad i=1, \ldots, n .
$$


Then from 1.2 and 1.3 we see that ${ }^{H} X_{(i)}$ and ${ }^{V} \theta^{(i)}$ have local expressions

$$
\begin{aligned}
& \tilde{e}_{(i)}={ }^{H} X_{(i)}=\frac{\partial}{\partial x^{i}}+\sum_{h} p_{a} \Gamma_{h i}^{a} \frac{\partial}{\partial x^{\bar{h}}}, \\
& \tilde{e}_{(\bar{\imath})}={ }^{V} \theta^{(i)}=\frac{\partial}{\partial x^{\bar{\imath}}} .
\end{aligned}
$$

We call the set $\left\{\tilde{e}_{(\alpha)}\right\}=\left\{\tilde{e}_{(i)}, \tilde{e}_{(\bar{\imath})}\right\}=\left\{{ }^{H} X_{(i)},{ }^{V} \theta^{(i)}\right\}$ the frame adapted to the Levi-Civita connection $\nabla_{g}$. The indices $\alpha, \beta, \ldots=1, \ldots, 2 n$ indicate the indices with respect to the adapted frame.

From equations 1.2$), 11.3,2.1)$ and $(2.2)$, we see that ${ }^{H} X$ and ${ }^{V} \omega$ have the components

$$
\begin{array}{ll}
{ }^{H} X=X^{i} \tilde{e}_{(i)}, \quad{ }^{H} X=\left({ }^{H} X^{\alpha}\right)=\left(\begin{array}{c}
X^{i} \\
0
\end{array}\right), \\
{ }_{\omega}=\sum_{i} \omega_{i} \tilde{e}_{(\bar{\imath})}, & V_{\omega}=\left({ }^{V} \omega^{\alpha}\right)=\left(\begin{array}{c}
0 \\
\omega_{i}
\end{array}\right)
\end{array}
$$

with respect to the adapted frame $\left\{\tilde{e}_{(\alpha)}\right\}$, where $X^{i}$ and $\omega_{i}$ are the local components of $X \in \Im_{0}^{1}\left(M^{n}\right)$ and $\omega \in \Im_{1}^{0}\left(M^{n}\right)$, respectively.

Let ${ }^{S} \nabla$ be the Levi-Civita connection determined by the Sasakian metric ${ }^{S} \mathrm{~g}$. The components of ${ }^{S} \nabla$ are given by [7]

$$
\begin{array}{rlrl}
{ }^{S} \Gamma_{j i}^{h} & =\Gamma_{j i}^{h}, & { }^{S} \Gamma_{\bar{j} \bar{\imath}}^{h}={ }^{S} \Gamma_{\bar{j}}^{\bar{h}}{ }_{i}={ }^{S} \Gamma_{\bar{j} \bar{\imath}}^{\bar{h}}=0, \\
{ }^{S} \Gamma_{j \bar{\imath}}^{h}=\frac{1}{2} p_{m} R_{. j .}^{h i m}, & { }^{S} \Gamma_{\bar{j} i}^{h}=\frac{1}{2} p_{m} R_{. i}^{h}{ }_{i j}{ }^{j m}, \\
{ }^{S} \Gamma_{j i}^{\bar{h}}=\frac{1}{2} p_{m} R_{j i h}{ }^{m}, & { }^{S} \Gamma_{j \bar{\imath}}^{\bar{h}}=-\Gamma_{j h}^{i}
\end{array}
$$

with respect to the adapted frame $\left\{\tilde{e}_{(\alpha)}\right\}$, where $R_{i j k}{ }^{h}$ are the local components of the curvature tensor $R$ of $\nabla_{g}$.

Let now $\tilde{X}, \tilde{Y} \in \Im_{0}^{1}\left(T^{*} M^{n}\right)$ and $\tilde{X}=\tilde{X}^{\alpha} \tilde{e}_{\alpha}, \tilde{Y}=\tilde{Y}^{\beta} \tilde{e}_{\beta}$. The covariant derivative ${ }^{S} \nabla_{\tilde{Y}} \tilde{X}$ along $\tilde{Y}$ has components

$$
{ }^{S} \nabla_{\tilde{Y}} \tilde{X}^{\alpha}=\tilde{Y}^{\gamma} \tilde{e}_{\gamma} \tilde{X}^{\alpha}+{ }^{S} \Gamma_{\gamma \beta}^{\alpha} \tilde{X}^{\beta} \tilde{Y}^{\gamma}
$$

with respect to the adapted frame $\left\{\tilde{e}_{(\alpha)}\right\}$.

Using (2.3)-2.6), we have

THEOREM 2.1. Let $M^{n}$ be a Riemannian manifold with metric $g$ and ${ }^{S} \nabla$ be the Levi-Civita connection of the cotangent bundle $T^{*} M^{n}$ equipped with the Sasakian metric ${ }^{S} g$. Then ${ }^{S} \nabla$ satisfies

(i) ${ }^{S} \nabla_{V_{\omega}}{ }^{V} \theta=0$,

(ii) ${ }^{S} \nabla_{V_{\omega}}{ }^{H} Y=\frac{1}{2}{ }^{H}\left(p\left(g^{-1} \circ R(, Y) \tilde{\omega}\right)\right)$,

(iii) ${ }^{S} \nabla_{H_{X}} V_{\theta}={ }^{V}\left(\nabla_{X} \theta\right)+\frac{1}{2}{ }^{H}\left(p\left(g^{-1} \circ R(, X) \tilde{\theta}\right)\right)$,

(iv) ${ }^{S} \nabla_{H_{X}}{ }^{H} Y={ }^{H}\left(\nabla_{X} Y\right)+\frac{1}{2} V(p R(X, Y))$ 
for all $X, Y \in \Im_{0}^{1}\left(M^{n}\right)$ and $\omega, \theta \in \Im_{1}^{0}\left(M^{n}\right)$, where $\tilde{\omega}=g^{-1} \circ \omega \in \Im_{0}^{1}\left(M^{n}\right)$, $R(, X) \tilde{\omega} \in \Im_{1}^{1}\left(M^{n}\right), g^{-1} \circ R(, X) \tilde{\omega} \in \Im_{0}^{2}\left(M^{n}\right)$.

3. Para-Nordenian structures on $\left(T^{*} M^{n},{ }^{S} g\right)$. An almost paracomplex manifold is an almost product manifold $\left(M^{n}, \varphi\right), \varphi^{2}=\mathrm{I}$, such that the two eigenbundles $T^{+} M^{n}$ and $T^{-} M^{n}$ associated to the two eigenvalues +1 and -1 of $\varphi$, respectively, have the same rank. Note that the dimension of an almost paracomplex manifold is necessarily even. Considering the paracomplex structure $\varphi$, we obtain the set $\{\mathrm{I}, \varphi\}$ on $M^{n}$, which is an isomorphic representation of the algebra of order 2 , called the algebra of paracomplex (or double) numbers and denoted by $R(j), j^{2}=1$.

A tensor field $\omega \in \Im_{q}^{0}\left(M^{2 n}\right)$ is said to be pure with respect to the paracomplex structure $\varphi$ if

$$
\omega\left(\varphi X_{1}, X_{2}, \ldots, X_{q}\right)=\omega\left(X_{1}, \varphi X_{2}, \ldots, X_{q}\right)=\cdots=\omega\left(X_{1}, X_{2}, \ldots, \varphi X_{q}\right)
$$

for any $X_{1}, X_{2}, \ldots, X_{q} \in \Im_{0}^{1}\left(M^{2 n}\right)$.

We define the operator $\phi_{\varphi}$ associated with $\varphi$ and applied to the pure tensor field $\omega$ by (see [10])

$$
\begin{array}{r}
\left(\phi_{\varphi} \omega\right)\left(Y, X_{1}, \ldots, X_{q}\right)=(\varphi Y)\left(\omega\left(X_{1}, \ldots, X_{q}\right)\right)-Y\left(\omega\left(\varphi X_{1}, X_{2}, \ldots, X_{q}\right)\right) \\
+\omega\left(\left(\mathrm{L}_{X_{1}} \varphi\right) Y, X_{2}, \ldots, X_{q}\right)+\cdots+\omega\left(X_{1}, X_{2}, \ldots,\left(\mathrm{L}_{X_{q}} \varphi\right) Y\right),
\end{array}
$$

where $\mathrm{L}_{X}$ denotes the Lie derivative with respect to $X$. We note that $\phi_{\varphi} \omega \in$ $\Im_{q+1}^{0}\left(M^{2 n}\right)$.

If $\phi_{\varphi} \omega=0$, then $\omega$ is said to be almost paraholomorphic with respect to the paracomplex algebra $R(j)$ (see [4], 8]).

A Riemannian manifold $\left(M^{2 n}, g\right)$ with an almost paracomplex structure $\varphi$ is said to be almost para-Nordenian if the Riemannian metric $g$ is pure with respect to $\varphi$. It is well known that the almost para-Nordenian manifold is para-Kähler $\left(\nabla_{g} \varphi=0\right)$ if and only if $g$ is paraholomorphic $\left(\phi_{\varphi} g=0\right)($ see $[8])$.

Let $\left(T^{*} M^{n},{ }^{S} g\right)$ be the cotangent bundle with the Sasakian metric ${ }^{S} g$. We define a tensor field $F$ of type $(1,1)$ on $T^{*} M^{n}$ by

$$
\left\{\begin{array}{l}
F^{H} X={ }^{V} \tilde{X}, \\
F^{V} \omega={ }^{H} \tilde{\omega}
\end{array}\right.
$$

for any $X \in \Im_{0}^{1}\left(M^{n}\right)$ and $\omega \in \Im_{1}^{0}\left(M^{n}\right)$, where $\tilde{X}=g \circ X \in \Im_{1}^{0}\left(M^{n}\right)$, $\tilde{\omega}=g^{-1} \circ \omega \in \Im_{0}^{1}\left(M^{n}\right)$. Then we obtain

$$
F^{2}=\text { I. }
$$


Indeed, by virtue of $(3.1)$ we have

$$
\begin{aligned}
F^{2}\left({ }^{H} X\right) & =F\left(F^{H} X\right)=F\left({ }^{V} \tilde{X}\right)={ }^{H} \tilde{\tilde{X}}={ }^{H} X, \\
F^{2}\left({ }^{V} \omega\right) & =F\left(F^{V} \omega\right)=F\left({ }^{H} \tilde{\omega}\right)={ }^{V} \tilde{\tilde{\omega}}={ }^{V} \omega
\end{aligned}
$$

for any $X \in \Im_{0}^{1}\left(M^{n}\right)$ and $\omega \in \Im_{1}^{0}\left(M^{n}\right)$, which implies $F^{2}=\mathrm{I}$.

Theorem 3.1. The triple $\left(T^{*} M^{n},{ }^{S} g, F\right)$ is an almost para-Nordenian manifold.

Proof. We put

$$
A(\tilde{X}, \tilde{Y})={ }^{S} g(F \tilde{X}, \tilde{Y})-{ }^{S} g(\tilde{X}, F \tilde{Y})
$$

for any $\tilde{X}, \tilde{Y} \in \Im_{0}^{1}\left(T^{*} M^{n}\right)$. From 1.4 1.6 and 3.1), we have

$$
\begin{aligned}
A\left({ }^{H} X,{ }^{H} Y\right) & ={ }^{S} g\left(F^{H} X,{ }^{H} Y\right)-{ }^{S} g\left({ }^{H} X, F^{H} Y\right) \\
& ={ }^{S} g\left({ }^{V} \tilde{X},{ }^{H} Y\right)-{ }^{S} g\left({ }^{H} X,{ }^{V} \tilde{Y}\right)=0, \\
A\left({ }^{H} X,{ }^{V} \omega\right) & ={ }^{S} g\left(F^{H} X,{ }^{V} \omega\right)-{ }^{S} g\left({ }^{H} X, F{ }^{V} \omega\right)={ }^{S} g\left({ }^{V} \tilde{X},{ }^{V} \omega\right)-{ }^{S} g\left({ }^{H} X,{ }^{H} \tilde{\omega}\right) \\
& =g^{-1}(g \circ X, \omega)-g\left(X, g^{-1} \circ \omega\right)=0, \\
A\left({ }^{V} \omega,{ }^{H} Y\right) & =-A\left({ }^{H} Y,{ }^{V} \omega\right)=0, \\
A\left({ }^{V} \omega,{ }^{V} \theta\right) & ={ }^{S} g\left(F^{V} \omega,{ }^{V} \theta\right)-{ }^{S} g\left({ }^{V} \omega, F^{V} \theta\right)=g^{-1}\left({ }^{H} \tilde{\omega},{ }^{V} \theta\right)-{ }^{S} g\left({ }^{V} \omega,{ }^{H} \tilde{\theta}\right)=0,
\end{aligned}
$$

i.e. ${ }^{S} g$ is pure with respect to $F$. Thus Theorem 3.1 is proved.

We now consider the covariant derivative of $F$. Taking into account (i)(iv) of Theorem 2.1 and (3.1), we obtain

$$
\begin{aligned}
\left({ }^{S} \nabla_{H_{X}} F\right)\left({ }^{H} Y\right)= & { }^{S} \nabla_{H_{X}}\left(F^{H} Y\right)-F\left({ }^{S} \nabla_{H_{X}}{ }^{H} Y\right) \\
= & { }^{S} \nabla_{H_{X}}{ }^{V} \tilde{Y}-F\left({ }^{S} \nabla_{H_{X}}{ }^{H} Y\right) \\
= & { }^{V}\left(\nabla_{X} \tilde{Y}\right)+\frac{1}{2}{ }^{H}\left(p\left(g^{-1} \circ R(, X) Y\right)\right) \\
& -F\left({ }^{H}\left(\nabla_{X} Y\right)+\frac{1}{2}{ }^{V}(p R(X, Y))\right) \\
= & \frac{1}{2}{ }^{H}\left(p g^{-1} \circ(R(, X) Y-R(X, Y))\right), \\
\left({ }^{S} \nabla_{V_{\omega}} F\right)\left({ }^{H} Y\right)= & { }^{S} \nabla_{V_{\omega}}\left(F^{H} Y\right)-F\left({ }^{S} \nabla_{V_{\omega}}{ }^{H} Y\right) \\
= & { }^{S} \nabla_{V_{\omega}}{ }^{V} \tilde{Y}-\frac{1}{2} F^{H}\left(p\left(g^{-1} \circ R(, Y) \tilde{\omega}\right)\right) \\
= & -\frac{1}{2}{ }^{V}(p R(, Y) \tilde{\omega}), \\
\left({ }^{S} \nabla_{H_{X}} F\right)\left({ }^{V} \theta\right)= & { }^{S} \nabla_{H_{X}}\left(F{ }^{V} \theta\right)-F\left({ }^{S} \nabla_{H^{H}}{ }^{V} \theta\right) \\
= & { }^{S} \nabla_{H_{X}}{ }^{H} \tilde{\theta}-F\left({ }^{V}\left(\nabla_{X} \theta\right)+\frac{1}{2}{ }^{H}\left(p\left(g^{-1} \circ R(, X) \tilde{\theta}\right)\right)\right) \\
= & { }^{H}\left(\nabla_{X} \tilde{\theta}\right)+\frac{1}{2}{ }^{V}(p R(X, \tilde{\theta}))-{ }^{H}\left(g^{-1} \circ\left(\nabla_{X} \theta\right)\right) \\
& -\frac{1}{2}{ }^{V}\left(p g \circ\left(g^{-1} \circ R(, X) \tilde{\theta}\right)\right) \\
= & \frac{1}{2}{ }^{V}(p R(X, \tilde{\theta})-p R(, X) \tilde{\theta}),
\end{aligned}
$$




$$
\begin{aligned}
\left({ }^{S} \nabla_{V_{\omega}} F\right)\left({ }^{V} \theta\right) & ={ }^{S} \nabla_{V_{\omega}}\left(F^{V_{\theta}}\right)-F\left({ }^{S} \nabla_{V_{\omega}}{ }^{V} \theta\right) \\
& ={ }^{S} \nabla_{V_{\omega}}{ }^{H} \tilde{\theta}=\frac{1}{2}{ }^{H}\left(p\left(g^{-1} \circ R(, \tilde{\theta}) \tilde{\omega}\right)\right) .
\end{aligned}
$$

From (3.2)-3.5) we have

THEOREM 3.2. The cotangent bundle of a Riemannian manifold is paraKählerian (paraholomorphic Nordenian) with respect to the metric ${ }^{S} g$ and almost paracomplex structure $F$ defined by (3.1) if and only if the Riemannian manifold is flat.

4. A necessary and sufficient condition for the complete lift of a vector field to be paraholomorphic. A vector field $\tilde{X} \in \Im_{0}^{1}\left(T^{*} M^{n}\right)$ with respect to which the almost para-Nordenian structure $F$ has a vanishing Lie derivative $\left(\mathrm{L}_{\tilde{X}} F=0\right)$ is said to be almost paraholomorphic (see [4]).

It is well known that [11, p. 277]

$$
\left\{\begin{array}{l}
{\left[{ }^{C} X,{ }^{H} Y\right]={ }^{H}[X, Y]+{ }^{V}\left(p\left(\mathrm{~L}_{X} \nabla\right) Y\right),} \\
{\left[{ }^{C} X,{ }^{C} \omega\right]={ }^{V}\left(\mathrm{~L}_{X} \omega\right),}
\end{array}\right.
$$

where $\left(\mathrm{L}_{X} \nabla\right) Y=\nabla_{Y} \nabla X+R(X, Y)$ and $\left(\mathrm{L}_{X} \nabla\right)(Y, Z)=\mathrm{L}_{X}\left(\nabla_{Y} X\right)-$ $\nabla_{Y}\left(\mathrm{~L}_{X} Z\right)-\nabla_{[X, Y]} Z$.

A vector field $X \in \Im_{0}^{1}\left(M^{n}\right)$ is called a Killing vector field (or infinitesimal isometry) if $\mathrm{L}_{X} g=0$, and $X$ is called an infinitesimal affine transformation if $\mathrm{L}_{X} \nabla_{g}=0$. A Killing vector field is necessarily an infinitesimal affine transformation, i.e. we have $\mathrm{L}_{X} \nabla_{g}=0$ as a consequence of $\mathrm{L}_{X} g=0$.

We now consider the Lie derivative of $F$ with respect to the complete lift ${ }^{C} X$. Taking account of (3.1) and 4.1), we obtain

$$
\begin{aligned}
\left(\mathrm{L}_{C} F\right)^{V} \theta & =\mathrm{L}_{C_{X}} F^{V} \theta-F\left(\mathrm{~L}_{C_{X}}{ }^{V} \theta\right)=\mathrm{L}_{C_{X}}{ }^{H} \tilde{\theta}-F\left({ }^{V}\left(\mathrm{~L}_{X} \theta\right)\right) \\
& =\mathrm{L}_{C_{X}}{ }^{H} \tilde{\theta}-{ }^{H}\left(g^{-1} \circ\left(\mathrm{L}_{X} \theta\right)\right) \\
& ={ }^{V}[X, \tilde{\theta}]+{ }^{V}\left(p\left(\mathrm{~L}_{X} \nabla\right) \tilde{\theta}\right)-{ }^{H}\left(g^{-1} \circ\left(\mathrm{L}_{X} \theta\right)\right) \\
& ={ }^{H}\left(\mathrm{~L}_{X}\left(g^{-1} \circ \theta\right)-g^{-1} \circ\left(\mathrm{L}_{X} \theta\right)\right)+{ }^{V}\left(p\left(\mathrm{~L}_{X} \nabla\right) \tilde{\theta}\right), \\
\left(\mathrm{L}_{C_{X}} F\right)^{H} Y & =\mathrm{L}_{C_{X}} F^{H} Y-F\left(\mathrm{~L}_{C_{X}}{ }^{H} Y\right) \\
& =\mathrm{L}_{C_{X}}{ }^{V} \tilde{Y}-F\left({ }^{H}[X, Y]+{ }^{V}\left(p\left(\mathrm{~L}_{X} \nabla\right)_{Y}\right)\right) \\
& ={ }^{V}\left(\mathrm{~L}_{X}(g \circ Y)-g \circ \mathrm{L}_{X} Y\right)-{ }^{H}\left(g^{-1} \circ p\left(\mathrm{~L}_{X} \nabla\right)_{Y}\right) .
\end{aligned}
$$

Let now $X$ be a Killing vector field $\left(\mathrm{L}_{X} g=0\right)$. Then by virtue of $\mathrm{L}_{X} \nabla=0$, from 4.2 and 4.3 we have $\mathrm{L}_{C_{X}} F=0$, i.e. ${ }^{C} X$ is paraholomorphic with respect to $F$. If we assume that $\mathrm{L}_{C_{X}} F=0$ and compute the equation 4.3 at $\left(x^{i}, 0\right), p_{i}=0$, then we get $\mathrm{L}_{X}(g \circ Y)=g \circ \mathrm{L}_{X} Y$. It follows that $\mathrm{L}_{X} g=0$. Hence, we have 
TheOREM 4.1. An infinitesimal transformation $X$ of the Riemannian manifold $\left(M^{n}, g\right)$ is a Killing vector field if and only if its complete lift ${ }^{C} X$ to the cotangent bundle $T^{*} M^{n}$ is an almost paraholomorphic vector field with respect to the almost para-Nordenian structure $\left(F,{ }^{S} g\right)$.

REMARK. Let ${ }^{R} \nabla \in \Im_{2}^{0}\left(T^{*} M^{n}\right)$ be a Riemannian extension of the connection $\nabla_{g}$ defined by (cf. [11, p. 268])

$$
{ }^{R} \nabla\left({ }^{C} X,{ }^{C} Y\right)=-p\left(\nabla_{X} Y+\nabla_{Y} X\right), \quad X, Y \in \Im_{0}^{1}\left(\mathrm{M}_{n}\right) .
$$

The metric ${ }^{R} \nabla$ has components

$$
{ }^{R} \nabla=\left(\begin{array}{cc}
-2 p_{a} \Gamma_{j i}^{a} & \delta_{i}^{j} \\
\delta_{j}^{i} & 0
\end{array}\right)
$$

with respect to the natural frame $\left\{\partial_{i}, \partial_{\bar{\imath}}\right\}$. From $(1.2),(1.3)$ and $(4.4)$ we easily see that

$$
{ }^{R} \nabla\left({ }^{H} X,{ }^{H} Y\right)=0, \quad{ }^{R} \nabla\left({ }^{V} \omega,{ }^{V} \theta\right)=0, \quad{ }^{R} \nabla\left({ }^{H} X,{ }^{V} \theta\right)={ }^{V}(\theta(X)),
$$

i.e. the metric ${ }^{R} \nabla$ is completely determined also by conditions 4.5. Using (1.6), (3.1) and 4.5), we have

$$
\begin{aligned}
\left({ }^{R} \nabla \circ F\right)\left({ }^{H} X,{ }^{H} Y\right) & ={ }^{R} \nabla\left(F^{H} X,{ }^{H} Y\right)={ }^{R} \nabla\left({ }^{V} \tilde{X},{ }^{H} Y\right)={ }^{V}(\tilde{X}(Y)) \\
& ={ }^{V}(g(X, Y))={ }^{S} g\left({ }^{H} X,{ }^{H} Y\right), \\
\left({ }^{R} \nabla \circ F\right)\left({ }^{H} X,{ }^{V} \theta\right) & ={ }^{R} \nabla\left(F^{H} X,{ }^{V} \theta\right)={ }^{R} \nabla\left({ }^{V} \tilde{X},{ }^{V} \theta\right)={ }^{S} g\left({ }^{H} X,{ }^{V} \theta\right)=0, \\
\left({ }^{R} \nabla \circ F\right)\left({ }^{V} \omega,{ }^{H} Y\right) & ={ }^{R} \nabla\left(F{ }^{V} \omega,{ }^{H} Y\right)={ }^{R} \nabla\left({ }^{H} \tilde{\omega},{ }^{H} Y\right)={ }^{S} g\left({ }^{V} \omega,{ }^{H} Y\right)=0, \\
\left({ }^{R} \nabla \circ F\right)\left({ }^{V} \omega,{ } \theta\right) & ={ }^{R} \nabla\left(F^{V} \omega,{ }^{V} \theta\right)={ }^{R} \nabla\left({ }^{H} \tilde{\omega},{ }^{V} \theta\right)={ }^{V}(\theta(\tilde{\omega})) \\
& ={ }^{V}\left(g^{-1}(\omega, \theta)\right)={ }^{S} g\left({ }^{V} \omega,{ }^{V} \theta\right),
\end{aligned}
$$

i.e. ${ }^{R} \nabla \circ F={ }^{S} g$. Thus the almost para-Nordenian structure $F$ determined by the condition 3.1 has an expression of the form $F=\left({ }^{R} \nabla\right)^{-1} \circ{ }^{S} g$.

Acknowledgements. This paper is supported by the Scientific and Technological Research Council of Turkey (TBAG-108T590).

\section{References}

[1] N. Cengiz and A. A. Salimov, Diagonal lift in the tensor bundle and its applications, Appl. Math. Comput. 142 (2003), 309-319.

[2] L. A. Cordero, C. T. J. Dodson and M. de León, Differential Geometry of Frame Bundles, Math. Appl. 47, Kluwer, Dordrecht, 1989.

[3] S. Gudmundsson and E. Kappos, On the geometry of tangent bundles, Expo. Math. 20 (2002), 1-41.

[4] G. I. Kruchkovich [G. I. Kručkovič], Hypercomplex structures on manifolds. I, Trudy Sem. Vektor. Tenzor. Anal. 16 (1972), 174-201 (in Russian). 
[5] K. P. Mok, On the differential geometry of frame bundles of Riemannian manifolds, J. Reine Angew. Math. 302 (1978), 16-31.

[6] A. A. Salimov and N. Cengiz, Lift of Riemannian metrics to tensor bundles, Izv. Vyssh. Uchebn. Zav. Mat. 2003, no. 11, 51-59 (in Russian); English transl.: Russian Math. (Iz. VUZ) 47 (2003), no. 11, 47-55.

[7] A. A. Salimov, A. Gezer and K. Akbulut, Geodesics of Sasakian metrics on tensor bundles, Mediterr. J. Math. 6 (2009), 135-147.

[8] A. A. Salimov, M. Iscan and F. Etayo, Paraholomorphic B-manifold and its properties, Topology Appl. 154 (2007), 925-933.

[9] S. Sasaki, On the differential geometry of tangent bundles of Riemannian manifolds, Tôhoku Math. J. 10 (1958), 338-354.

[10] K. Yano and M. Ako, On certain operators associated with tensor fields, Kōdai Math. Sem. Rep. 20 (1968), 414-436.

[11] K. Yano and S. Ishihara, Tangent and Cotangent Bundles. Differential Geometry, Pure Appl. Math. 16, Dekker, New York, 1973.

Arif A. Salimov

Department of Mathematics

Science Faculty

Atatürk University

TR-25240, Erzurum, Turkey

and

Department of Algebra and Geometry

Faculty of Mechanics and Mathematics

Baku State University

AZ-1148, Baku, Azerbaijan

E-mail: asalimov@atauni.edu.tr
Filiz Agca

Department of Mathematics Karadeniz Technical University

TR-61080 Trabzon, Turkey

E-mail: filiz_math@hotmail.com

Received 2.12.2009

and in final form 12.1.2010 